$\mathrm{J}$ o u r n a l of

Mathematics

and Applications

JMA No 42, pp 117-133 (2019)

\title{
The Existence of Monotonic Solutions of a Class of Quadratic Integral Equations of Volterra Type
}

\author{
Osman Karakurt and Ömer Faruk Temizer*
}

\begin{abstract}
Using the technique associated with measure of noncompactness we prove the existence of monotonic solutions of a class of quadratic integral equation of Volterra type in the Banach space of real functions defined and continuous on a bounded and closed interval.
\end{abstract}

AMS Subject Classification: 45D05, 45M20.

Keywords and Phrases: Nonlinear Volterra integral equations; Measure of noncompactness; Fixed point theorem.

\section{Introduction}

The theory of integral operators and integral equations is an important part of nonlinear analysis. This theory is frequently applicable in other branches of mathematics and mathematical physics, engineering, economics, biology as well in describing problems connected with real world $[1,2,7,9,10,11]$.

The aim of this paper is to investigate the existence of nondecreasing solutions of a class of a quadratic integral equations of Volterra type. We will look for solutions of those equations in the Banach space of real functions being defined and continuous on a bounded and closed interval. The main tool used in our investigation is the technique of measure of noncompactness which is frequently used in several branches of nonlinear analysis [4, 7, 5, 9].

We will apply the measure of noncompactness defined in [6] to proving the solvability of the considered equations in the class of monotonic functions.

The results of this paper generalize the results obtained earlier in the paper [3].

COPYRIGHT (C) by Publishing House of Rzeszów University of Technology P.O. Box 85, 35-959 Rzeszów, Poland 


\section{Notation and auxiliary facts}

Now, we are going to recall the basic results which are needed further on.

Assume that $E$ is a real Banach space with the norm $\|$.$\| and the zero element 0$. Denote by $B(x, r)$ the closed ball centered at $x$ and with radius $r$ and by $B_{r}$ the ball $B(0, r)$. If $X$ is a nonempty subset of $E$ we denote by $\bar{X}, \operatorname{Conv} X$ the closure and the convex closure of $X$, respectively.

With the symbols $\lambda X$ and $X+Y$ we denote the algebraic operations on the sets. Finally, let us denote by $\mathfrak{M}_{E}$ the family of all nonempty and bounded subsets of $E$ and by $\mathfrak{N}_{E}$ its subfamily consisting of all relatively compact sets.

Definition 2.1 (See [4]). A function $\mu: \mathfrak{M}_{E} \rightarrow[0, \infty)$ is said to be a measure of noncompactness in the space $E$ if it is satisfies the following conditions

(1) The family $\operatorname{ker} \mu=\left\{\mathrm{X} \in \mathfrak{M}_{\mathrm{E}}: \mu(\mathrm{X})=0\right\} \neq \emptyset$ and $\operatorname{ker} \mu \subset \mathfrak{N}_{\mathrm{E}}$,

(2) $X \subset Y \Rightarrow \mu(X) \leq \mu(Y)$,

(3) $\mu(\bar{X})=\mu(\operatorname{Conv} X)=\mu(X)$,

(4) $\mu(\lambda X+(1-\lambda) Y) \leq \lambda \mu(X)+(1-\lambda) \mu(Y)$, for $\lambda \in[0,1]$,

(5) If $\left\{X_{n}\right\}_{n}$ is a sequence of closed sets from $\mathfrak{M}_{E}$ such that $X_{n+1} \subset X_{n}$ for $n=1,2, \ldots$ and if $\lim _{n \rightarrow \infty} \mu\left(X_{n}\right)=0$, then the set $X_{\infty}=\bigcap_{n=1}^{\infty} X_{n}$ is nonempty.

The family ker $\mu$ described above is called the kernel of the measure of noncompactness $\mu$. Further facts concerning measures of noncompactness and their properties may be found in [4].

Now, let us suppose that Q is a nonempty subset of the Banach space $E$ and the operator $F: \mathrm{Q} \rightarrow E$ is continuous and transforms bounded sets onto bounded ones. We say that $F$ satisfies the Darbo condition (with a constant $k \geq 0$ ) with respect to a measure of noncompactness $\mu$ if for any bounded subset $X$ of $Q$ the following inequality holds:

$$
\mu(F X) \leq k \mu(X) .
$$

If $F$ satisfies the Darbo condition with $k<1$ then it is said to be a contraction with respect to $\mu,[8]$. For our further purposes we will only need the following fixed point theorem.

Theorem 2.1. Let $Q$ be a nonempty, bounded, closed and convex subset of the Banach space $E$ and $\mu$ be a measure of noncompactness in $E$. Let $F: Q \rightarrow Q$ be a continuous transformation such that $\mu(F X) \leq k \mu(X)$ for any nonempty subset $X$ of $Q$, where $k \in[0,1)$ is a constant. Then, $F$ has a fixed point in the set $Q,[3]$.

Remark 1. Under assumptions of the above theorem it can be shown that, the set Fix $F$ of fixed points of $F$ belonging to $Q$ is a member of ker $\mu$. This observation allows us to characterize solutions of considered equations, [3]. 
In what follows, we will work in the classical Banach space $C[0, M]$ consisting of all real functions defined and continuous on the interval $[0, \mathrm{M}]$. For convenience, we write $I=[0, M]$ and $C(I)=C[0, M]$. The space $C(I)$ is furnished by the standard norm $\|x\|=\max \{|x(t)|: t \in I\}$.

Now, we recall the definition of a measure of noncompactness in $C(I)$ which will be used in the sequel. That measure was introduced and studied in the paper [6].

To do this let us fix a nonempty and bounded subset $X$ of $C(I)$. For $\varepsilon>0$ and $x \in X$ denote by $w(x, \varepsilon)$ the modulus of continuity of $x$ defined by

$$
w(x, \varepsilon)=\sup \{|x(t)-x(s)|: t, s \in I,|t-s| \leq \varepsilon\} .
$$

Further, let us put

$$
\begin{gathered}
w(X, \varepsilon)=\sup \{w(x, \varepsilon): x \in X\}, \\
w_{0}(X)=\lim _{\varepsilon \rightarrow 0} w(X, \varepsilon) .
\end{gathered}
$$

Next, let us define the following quantities

$$
\begin{gathered}
i(x)=\sup \{|x(s)-x(t)|-[x(s)-x(t)]: t, s \in I, t \leq s\}, \\
i(X)=\sup \{i(x): x \in X\} .
\end{gathered}
$$

Observe that, $i(X)=0$ if and only if all functions belonging to $X$ are nondecreasing on $I$. Finally, let us put

$$
\mu(X)=w_{0}(X)+i(X)
$$

It can be shown that, the function $\mu$ is a measure of noncompactness in the space $C(I)$ (see [6]). Moreover, the kernel ker $\mu$ consist of all sets $X$ belonging to $\mathfrak{M}_{C(I)}$ such that all functions from $X$ are equicontinuous and nondecreasing on the interval $I$.

\section{Main result}

In this section, we apply the above defined measure of noncompactness $\mu$ to the study of monotonic solutions of our integral equation.

We consider the following nonlinear integral equation of Volterra type

$$
x(t)=a(\alpha(t))+(T x)(\beta(t)) \int_{0}^{\gamma(t)} v(t, \tau, x(\eta(\tau))) d \tau, \quad t \in I=[0, M] .
$$

The functions $a(\alpha(t)), v(t, \tau, x(\eta(\tau)))$ and $(T x)(\beta(t))$ appearing in this equation are given while $x=x(t)$ is an unknown function. This equation will be examined under the following assumptions: 
(i) $\alpha, \beta, \gamma, \eta: I \rightarrow I$ are continuous functions and $\alpha, \beta, \gamma$ are nondecreasing on $I$.

(ii) The function $a \in C(I)$ is nondecreasing and nonnegative on the interval $I$.

(iii) $v: I \times I \times \mathbb{R} \rightarrow \mathbb{R}$ is a continuous function such that $v: I \times I \times \mathbb{R}_{+} \rightarrow \mathbb{R}_{+}$and for arbitrarily fixed $\tau \in I$ and $x \in \mathbb{R}_{+}$the function $t \rightarrow v(t, \tau, x)$ is nondecreasing on $I$.

(iv) There exists a nondecreasing function $f: \mathbb{R}_{+} \rightarrow \mathbb{R}_{+}$such that the inequality $|v(t, \tau, x)| \leq f(|x|)$ holds for $t, \tau \in I$ and $x \in \mathbb{R}$.

$(v)$ The operator $T: C(I) \rightarrow C(I)$ is continuous and $T$ is a positive operator, i.e. $T x \geq 0$ if $x \geq 0$.

(vi) There exist nonnegative constants $c, d$ and $p>0$ such that $|(T x)(t)| \leq c+d\|x\|^{p}$ for each $x \in C(I)$ and all $t \in I$.

(vii) The inequality $a(\|\alpha\|)+\left(c+d r^{p}\right) M f(r) \leq r$ has a positive solution $r_{0}$.

(viii) The operator $T$ in $B_{r_{0}}^{+}=\left\{x \in B_{r_{0}}: x(t) \geq 0, t \in I\right\}$ satisfies the inequality $\mu(T X) \leq \theta \mu(X)$ for the measure of noncompactness $\mu$ with a constant $\theta$ such that $M f\left(r_{0}\right) \theta<1$, where $\theta \in[0,1)$.

Then, we have the following theorem:

Theorem 3.1. Under the assumptions (i)-(viii) the equation (3.1) has at least one solution $x=x(t)$ which belongs to the space $C(I)$ and is nondecreasing on the interval $I$.

Proof. Let us consider the operator $V$ defined on the space $C(I)$ in the following way:

$$
(V x)(t)=a(\alpha(t))+(T x)(\beta(t)) \int_{0}^{\gamma(t)} v(t, \tau, x(\eta(\tau))) d \tau .
$$

The proof will now proceed in two steps: firstly $V$ is continuous and secondly $V$ is contraction transformation on $B_{r_{0}}^{+} \subset C(I)$.

Step 1. In view of the assumptions $(i),(i i),(i i i)$ and $(v)$ it follows that, the function $V x$ is continuous on $I$ for any function $x \in C(I)$, i.e., $V$ transforms the space $C(I)$ into itself. Moreover, keeping in mind the assumptions (iv) and (vi) we get

$$
\begin{aligned}
|(V x)(t)| & \leq|a(\alpha(t))|+|(T x)(\beta(t))| \int_{0}^{\gamma(t)} v(t, \tau, x(\eta(\tau))) d \tau \mid \\
& \leq a(\|\alpha\|)+\left(c+d\|x\|^{p}\right) \int_{0}^{\gamma(t)} f(|x(\eta(\tau))|) d \tau \\
& \leq a(\|\alpha\|)+\left(c+d\|x\|^{p}\right) \int_{0}^{\gamma(t)} f(\|x\|) d \tau \\
& \leq a(\|\alpha\|)+\left(c+d\|x\|^{p}\right) M f(\|x\|) .
\end{aligned}
$$


Hence, we obtain the inequality

$$
\|V x\| \leq a(\|\alpha\|)+\left(c+d\|x\|^{p}\right) M f(\|x\|) .
$$

For $r_{0} \geq\|x\|$ such that provide assumption (vii), we get $\|V x\| \leq r_{0}$. This shows that $V$ transforms the ball $B_{r_{0}}$ into itself i.e., $V: B_{r_{0}} \rightarrow B_{r_{0}}$.

Let us consider the operator $V$ on the subset $B_{r_{0}}^{+}$of the ball $B_{r_{0}}$ defined by

$$
B_{r_{0}}^{+}=\left\{x \in B_{r_{0}}: x(t) \geq 0, \quad t \in I\right\} .
$$

Since the function $x$ defined as $x(t)=r_{0}$ for all $t \in I$ is a member of the set $B_{r_{0}}^{+}$, the set $B_{r_{0}}^{+}$is nonempty. Since $B_{r_{0}}$ is bounded, $B_{r_{0}}^{+}$is bounded. The inequalities

$$
\lambda x(t)+(1-\lambda) y(t) \geq 0
$$

and

$$
\|\lambda x+(1-\lambda) y\| \leq \lambda\|x\|+(1-\lambda)\|y\| \leq \lambda r_{0}+(1-\lambda) r_{0}=r_{0}
$$

hold for all $x, y \in B_{r_{0}}^{+}, t \in I$ and $\lambda$ such that $0 \leq \lambda \leq 1$. So, $B_{r_{0}}^{+}$is convex.

Let us take a convergent sequence $\left(x_{n}\right) \subset B_{r_{0}}^{+} \subset B_{r_{0}}$ so that $\lim _{n \rightarrow \infty} x_{n}=x$. Since

$$
\left\|x_{n}-x\right\|=\max _{t \in I}\left|x_{n}(t)-x(t)\right| \rightarrow 0 \quad(n \rightarrow \infty),
$$

we get $\lim _{n \rightarrow \infty} x_{n}(t)=x(t)$. Hence, we have $x(t) \geq 0$ for all $t \in I$. Thus, $x \in B_{r_{0}}^{+}$ and $B_{r_{0}}^{+}$is closed.

In view of these facts and assumptions $(i),(i i),(i i i)$ and $(v)$ it follows that $V$ transforms the set $B_{r_{0}}^{+}$into itself.

Now, we show that $V$ is continuous on the set $B_{r_{0}}^{+}$. To do this let us fix $\varepsilon>0$ and take arbitrarily $x, y \in B_{r_{0}}^{+}$such that $\|x-y\| \leq \varepsilon$. Then, for $t \in I$ we get the following inequalities:

$$
\begin{aligned}
& |(V x)(t)-(V y)(t)| \\
= & \left|(T x)(\beta(t)) \int_{0}^{\gamma(t)} v(t, \tau, x(\eta(\tau))) d \tau-(T y)(\beta(t)) \int_{0}^{\gamma(t)} v(t, \tau, y(\eta(\tau))) d \tau\right| \\
\leq & \left|(T x)(\beta(t)) \int_{0}^{\gamma(t)} v(t, \tau, x(\eta(\tau))) d \tau-(T y)(\beta(t)) \int_{0}^{\gamma(t)} v(t, \tau, x(\eta(\tau))) d \tau\right| \\
+ & \left|(T y)(\beta(t)) \int_{0}^{\gamma(t)} v(t, \tau, x(\eta(\tau))) d \tau-(T y)(\beta(t)) \int_{0}^{\gamma(t)} v(t, \tau, y(\eta(\tau))) d \tau\right|
\end{aligned}
$$




$$
\begin{aligned}
& \leq|(T x)(\beta(t))-(T y)(\beta(t))| \int_{0}^{\gamma(t)}|v(t, \tau, x(\eta(\tau)))| d \tau \\
& +|(T y)(\beta(t))| \int_{0}^{\gamma(t)}|v(t, \tau, x(\eta(\tau)))-v(t, \tau, y(\eta(\tau)))| d \tau \\
& \leq|(T x)(\beta(t))-(T y)(\beta(t))| \int_{0}^{\gamma(t)} f(|x(\eta(\tau))|) d \tau \\
& +|(T y)(\beta(t))| \int_{0}^{\gamma(t)}|v(t, \tau, x(\eta(\tau)))-v(t, \tau, y(\eta(\tau)))| d \tau \\
& \leq|(T x)(\beta(t))-(T y)(\beta(t))| \int_{0}^{\gamma(t)} f(\|x\|) d \tau \\
& +|(T y)(\beta(t))| \int_{0}^{\gamma(t)}|v(t, \tau, x(\eta(\tau)))-v(t, \tau, y(\eta(\tau)))| d \tau \\
& \leq|(T x)(\beta(t))-(T y)(\beta(t))| \int_{0}^{\gamma(t)} f\left(r_{0}\right) d \tau \\
& +|(T y)(\beta(t))| \int_{0}^{\gamma(t)}|v(t, \tau, x(\eta(\tau)))-v(t, \tau, y(\eta(\tau)))| d \tau \\
& \leq|(T x-T y)(\beta(t))| \int_{0}^{\gamma(t)} f\left(r_{0}\right) d \tau \\
& +\left(c+d\|y\|^{p}\right) \int_{0}^{\gamma(t)}|v(t, \tau, x(\eta(\tau)))-v(t, \tau, y(\eta(\tau)))| d \tau \\
& \leq\|T x-T y\| \int_{0}^{\gamma(t)} f\left(r_{0}\right) d \tau+\left(c+d r_{0}^{p}\right) \int_{0}^{\gamma(t)} \beta_{r_{0}}(\varepsilon) d \tau \\
& \leq\|T x-T y\| M f\left(r_{0}\right)+\left(c+d r_{0}^{p}\right) \beta_{r_{0}}(\varepsilon) M,
\end{aligned}
$$

where $\beta_{r_{0}}(\varepsilon)$ is defined as

$$
\beta_{r_{0}}(\varepsilon)=\sup \left\{|v(t, \tau, x)-v(t, \tau, y)|: t, \tau \in I, x, y \in\left[0, r_{0}\right],|x-y| \leq \varepsilon\right\} .
$$

From the above estimate we obtain the following inequality:

$$
\|V x-V y\| \leq\|T x-T y\| M f\left(r_{0}\right)+\left(c+d r_{0}^{p}\right) M \beta_{r_{0}}(\varepsilon) .
$$

From the uniform cotinuity of the function $v$ on the set $I \times I \times\left[0, r_{0}\right]$ we have that $\beta_{r_{0}}(\varepsilon) \rightarrow 0$ as $\varepsilon \rightarrow 0$ and from the continuity of $T$, we have that $\|T x-T y\| \rightarrow 0$ as $\varepsilon \rightarrow 0$. The last inequality implies continuity of the operator $V$ on the set $B_{r_{0}}^{+}$.

Step 2. In what follows let us take a nonempty set $X \subset B r_{0}^{+}$. Further, fix arbitrarily a number $\varepsilon>0$ and choose $x \in X$ and $t, s \in[0, M]$ such that $|t-s| \leq \varepsilon$. Without loss of generality we may assume that $t \leq s$. Then, in view of our assumptions we obtain 


$$
\begin{aligned}
& |(V x)(s)-(V x)(t)| \\
\leq & |a(\alpha(s))-a(\alpha(t))| \\
+ & \left|(T x)(\beta(s)) \int_{0}^{\gamma(s)} v(s, \tau, x(\eta(\tau))) d \tau-(T x)(\beta(t)) \int_{0}^{\gamma(t)} v(t, \tau, x(\eta(\tau))) d \tau\right| \\
\leq & w(a, w(\alpha, \varepsilon))+\left|[(T x)(\beta(s))-(T x)(\beta(t))] \int_{0}^{\gamma(s)} v(s, \tau, x(\eta(\tau))) d \tau\right| \\
+ & \left|(T x)(\beta(t)) \int_{0}^{\gamma(s)} v(s, \tau, x(\eta(\tau))) d \tau-(T x)(\beta(t)) \int_{0}^{\gamma(s)} v(t, \tau, x(\eta(\tau))) d \tau\right| \\
+ & \left|(T x)(\beta(t)) \int_{0}^{\gamma(s)} v(t, \tau, x(\eta(\tau))) d \tau-(T x)(\beta(t)) \int_{0}^{\gamma(t)} v(t, \tau, x(\eta(\tau))) d \tau\right| \\
\leq & w(a, w(\alpha, \varepsilon))+|(T x)(\beta(s))-(T x)(\beta(t))| \int_{0}^{\gamma(s)}|v(s, \tau, x(\eta(\tau)))| d \tau \\
+ & |(T x)(\beta(t))| \int_{0}^{\gamma(s)}|v(s, \tau, x(\eta(\tau)))-v(t, \tau, x(\eta(\tau)))| d \tau \\
+ & \mid(T x)\left(\beta(t)\left|\int_{\gamma(t)}^{\gamma(s)} v(t, \tau, x(\eta(\tau))) d \tau\right|\right. \\
+ & \left(c+d r_{0}^{p}\right) f\left(r_{0}\right)|\gamma(s)-\gamma(t)| \\
\leq & w(a, w(\alpha, \varepsilon))+w(T x, w(\beta, \varepsilon)) M f\left(r_{0}\right)+\left(c+d r_{0}^{p}\right) M \xi_{r_{0}}(\varepsilon) \\
+ & w(a, w(\alpha, \varepsilon))+w(T x, w(\beta, \varepsilon)) \int_{0}^{\gamma(s)} f\left(r_{0}\right) d \tau+\left(c+d r_{0}^{p}\right) \int_{0}^{\gamma(s)} \xi_{r_{0}}(\varepsilon) d \tau
\end{aligned}
$$

where $\xi_{r_{0}}(\varepsilon)$ is defined as

$$
\left.\left.\xi_{r_{0}}(\varepsilon)=\sup \{\mid v(s, \tau, x))-v(t, \tau, x)\right)|: t, s, \tau \in I,| s-t \mid \leq \varepsilon, x \in\left[0, r_{0}\right]\right\} .
$$

Notice, that in view of the uniform continuity of the function $v$ on the set $I \times I \times\left[0, r_{0}\right]$ and from the uniform continuity of the function $\gamma$ on the interval $I$, we have $\xi_{r_{0}}(\varepsilon) \rightarrow 0$ as $\varepsilon \rightarrow 0$ and $(\gamma(s)-\gamma(t)) \rightarrow 0$. Thus, we have the inequality

$$
\begin{aligned}
& |(V x)(s)-(V x)(t)| \\
\leq & w(a, w(\alpha, \varepsilon))+w(T x, w(\beta, \varepsilon)) M f\left(r_{0}\right)+\left(c+d r_{0}^{p}\right) M \xi_{r_{0}}(\varepsilon) \\
+ & \left(c+d r_{0}^{p}\right) f\left(r_{0}\right)|\gamma(s)-\gamma(t)| .
\end{aligned}
$$

If we take the supremum at this inequality over the $t$ 's and $s$ 's, we have the inequality

$$
\begin{aligned}
w(V x, \varepsilon) & \leq w(a, w(\alpha, \varepsilon))+w(T x, w(\beta, \varepsilon)) M f\left(r_{0}\right)+\left(c+d r_{0}^{p}\right) M \xi_{r_{0}}(\varepsilon) \\
& +\left(c+d r_{0}^{p}\right) f\left(r_{0}\right) w(\gamma, \varepsilon) .
\end{aligned}
$$


If we take the supremum at this inequality over $x$ 's, we have the following estimation

$$
\begin{aligned}
w(V X, \varepsilon) & \leq w(a, w(\alpha, \varepsilon))+w(T X, w(\beta, \varepsilon)) M f\left(r_{0}\right)+\left(c+d r_{0}^{p}\right) M \xi_{r_{0}}(\varepsilon) \\
& +\left(c+d r_{0}^{p}\right) f\left(r_{0}\right) w(\gamma, \varepsilon) .
\end{aligned}
$$

For $\varepsilon \rightarrow 0$, we have

$$
w_{0}(V X) \leq M f\left(r_{0}\right) w_{0}(T X) .
$$

On the other hand, let us fix arbitrarily $x \in X$ and $t, s \in I$ such that $t \leq s$. Then, we have the following estimate:

$$
\begin{aligned}
& |(V x)(s)-(V x)(t)|-[(V x)(s)-(V x)(t)] \\
= & \mid a(\alpha(s))+(T x)(\beta(s)) \int_{0}^{\gamma(s)} v(s, \tau, x(\eta(\tau))) d \tau \\
- & a(\alpha(t))-(T x)(\beta(t)) \int_{0}^{\gamma(t)} v(t, \tau, x(\eta(\tau))) d \tau \mid \\
- & {\left[a(\alpha(s))+(T x)(\beta(s)) \int_{0}^{\gamma(s)} v(s, \tau, x(\eta(\tau))) d \tau\right.} \\
- & \left.a(\alpha(t))-(T x)(\beta(t)) \int_{0}^{\gamma(t)} v(t, \tau, x(\eta(\tau))) d \tau\right] \\
\leq & {[|a(\alpha(s))-a(\alpha(t))|-(a(\alpha(s))-a(\alpha(t)))] } \\
+ & \left|(T x)(\beta(s)) \int_{0}^{\gamma(s)} v(s, \tau, x(\eta(\tau))) d \tau-(T x)(\beta(t)) \int_{0}^{\gamma(t)} v(t, \tau, x(\eta(\tau))) d \tau\right| \\
- & {\left[(T x)(\beta(s)) \int_{0}^{\gamma(s)} v(s, \tau, x(\eta(\tau))) d \tau-(T x)(\beta(t)) \int_{0}^{\gamma(t)} v(t, \tau, x(\eta(\tau))) d \tau\right] } \\
\leq & \left|(T x)(\beta(s)) \int_{0}^{\gamma(s)} v(s, \tau, x(\eta(\tau))) d \tau-(T x)(\beta(t)) \int_{0}^{\gamma(s)} v(s, \tau, x(\eta(\tau))) d \tau\right| \\
+ & \left|(T x)(\beta(t)) \int_{0}^{\gamma(s)} v(s, \tau, x(\eta(\tau))) d \tau-(T x)(\beta(t)) \int_{0}^{\gamma(t)} v(t, \tau, x(\eta(\tau))) d \tau\right| \\
- & {\left[(T x)(\beta(s)) \int_{0}^{\gamma(s)} v(s, \tau, x(\eta(\tau))) d \tau-(T x)(\beta(t)) \int_{0}^{\gamma(s)} v(s, \tau, x(\eta(\tau))) d \tau\right] } \\
- & {\left[(T x)(\beta(t)) \int_{0}^{\gamma(s)} v(s, \tau, x(\eta(\tau))) d \tau-(T x)(\beta(t)) \int_{0}^{\gamma(t)} v(t, \tau, x(\eta(\tau))) d \tau\right] } \\
\leq & {[|(T x)(\beta(s))-(T x)(\beta(t))|-[(T x)(\beta(s))-(T x)(\beta(t))]] \int_{0}^{\gamma(s)} v(s, \tau, x(\eta(\tau))) d \tau } \\
\leq & {[|(T x)(\beta(s))-(T x)(\beta(t))|-[(T x)(\beta(s))-(T x)(\beta(t))]] M f\left(r_{0}\right) . }
\end{aligned}
$$

If we take supremum on both sides of this inequality over the $t, s \in I=[0, M]$, we 
have the inequality

$$
\begin{aligned}
i(V x) & \leq M f\left(r_{0}\right) \sup [|(T x)(\beta(s))-(T x)(\beta(t))|-[(T x)(\beta(s))-(T x)(\beta(t))]] \\
& \leq M f\left(r_{0}\right) i(T x),
\end{aligned}
$$

since the function $\beta$ is nondecreasing. If we take supremum over the $x$ 's, we get the inequality

$$
i(V X) \leq M f\left(r_{0}\right) i(T X) .
$$

Finally, from the inequalities (3.2) and (3.3), we obtain

$$
\mu(V X) \leq M f\left(r_{0}\right) \mu(T X) \leq M f\left(r_{0}\right) \theta \mu(X) .
$$

From the assumption (viii) which is

$$
M f\left(r_{0}\right) \theta<1
$$

and by applying Theorem 2.1, $V$ has a fixed point in the set $B_{r_{0}}^{+}$.

Let us remember that from Remark 1, the set Fix $V$ of fixed points of $V$ belonging to $C(I)$ is a member of $\operatorname{ker} \mu$. i.e. $\mu(\operatorname{Fix} V)=0$ and this implies $i(\operatorname{Fix} V)=0$. Therefore the solutions are nondecreasing on $I$. Thus the proof is completed.

Corollary 3.1. We assume that the function a is positive, the function $f$ is continuous and the assumptions (i)-(vi) and (viii) are provided in the Theorem 3.1. Let us take the inequality

$$
a(\|\alpha\|)+(c+d) M f(1)<1
$$

instead of (vii). So, the function $h$ defined as

$$
h:[0,1] \rightarrow \mathbb{R}, h(r)=a(\|\alpha\|)+\left(c+d r^{p}\right) M f(r)-r
$$

is continuous and

$$
h(0)=a(\|\alpha\|)+c M f(0)>0
$$

and

$$
h(1)=a(\|\alpha\|)+(c+d) M f(1)-1<0 .
$$

Thus, there exists at least one a number $r_{0} \in(0,1)$ such that $h\left(r_{0}\right)=0$. Consequently, all of the assumptions of the Theorem 3.1 hold and the equation (3.1) has at least one solution $x=x(t) \in B_{r_{0}}^{+}$.

Example 3.1. Let us consider the equation

$$
x(t)=\frac{t^{2}}{5}+\frac{1+x^{2}(t)}{2} \int_{0}^{t^{2}} \frac{\sin t+e^{x\left(\tau^{2}\right)}}{8+\tau} d \tau, \quad t \in I=[0,1],
$$


where $\alpha(t)=t^{2}, \beta(t)=t, \gamma(t)=t^{2}, \eta(\tau)=\tau^{2}, a(s)=\frac{s}{5}, a(\alpha(t))=\frac{t^{2}}{5}$ and the function $a$ is nondecreasing and positive and $\|\alpha\|=1, a(\|\alpha\|)=\frac{1}{5}$.

$$
v(t, \tau, x)=\frac{\sin t+e^{x}}{8+\tau}
$$

and

$$
(T x)(t)=\frac{1+x^{2}(t)}{2} .
$$

We have the following estimate

$$
|v(t, \tau, x)|=\left|\frac{\sin t+e^{x}}{8+\tau}\right| \leq \frac{1+e^{x}}{8} \leq \frac{1+e^{|x|}}{8}=f(|x|)
$$

for all $t, \tau \in I$ and $x \in \mathbb{R}$. From the above equation we see that $f(x)=\frac{1+e^{x}}{8}$. Let us see that the operator $T$ is continuous. Let $x_{0}$ be arbitrarily element chosen from $C(I)$. For $\left\|x-x_{0}\right\|<\delta$, we have the following estimate:

$$
\begin{aligned}
\left\|T x-T x_{0}\right\| & =\max _{t \in I}\left|\frac{1+x^{2}(t)}{2}-\frac{1+x_{0}^{2}(t)}{2}\right| \\
& =\frac{1}{2} \max _{t \in I}\left|x^{2}(t)-x_{0}^{2}(t)\right| \\
& =\frac{1}{2} \max _{t \in I}\left[\left|x(t)-x_{0}(t) \| x(t)+x_{0}(t)\right|\right]
\end{aligned}
$$

and

$$
|x(t)|=\left|x(t)-x_{0}(t)+x_{0}(t)\right| \leq\left|x(t)-x_{0}(t)\right|+\left|x_{0}(t)\right| \leq\left\|x-x_{0}\right\|+\left\|x_{0}\right\|
$$

such that,

$$
|x(t)| \leq \delta+\left\|x_{0}\right\|
$$

From the inequality (3.5), we obtain

$$
\left|x(t)+x_{0}(t)\right| \leq|x(t)|+\left\|x_{0}\right\| \leq \delta+2\left\|x_{0}\right\| .
$$

Thus, we obtain

$$
\begin{aligned}
\left\|T x-T x_{0}\right\| & =\frac{1}{2} \max _{t \in I}\left[\left|x(t)-x_{0}(t) \| x(t)+x_{0}(t)\right|\right] \\
& \leq \frac{1}{2}\left(\delta+2\left\|x_{0}\right\|\right) \max _{t \in I}\left|x(t)-x_{0}(t)\right| \\
& =\frac{1}{2}\left(\delta+2\left\|x_{0}\right\|\right)\left\|x-x_{0}\right\| .
\end{aligned}
$$

Taking

$$
\frac{1}{2}\left(\delta+2\left\|x_{0}\right\|\right) \delta=\varepsilon
$$


we get

$$
\begin{aligned}
\delta^{2}+2\left\|x_{0}\right\| \delta-2 \varepsilon=0 & \Rightarrow\left(\delta+\left\|x_{0}\right\|\right)^{2}-\left\|x_{0}\right\|^{2}-2 \varepsilon=0 \\
& \Rightarrow\left(\delta+\left\|x_{0}\right\|\right)^{2}=\left\|x_{0}\right\|^{2}+2 \varepsilon \\
& \Rightarrow \delta+\left\|x_{0}\right\|=\sqrt{\left\|x_{0}\right\|^{2}+2 \varepsilon}
\end{aligned}
$$

If $\delta$ is chosen as

$$
\delta=\sqrt{\left\|x_{0}\right\|^{2}+2 \varepsilon}-\left\|x_{0}\right\|>0
$$

it is seen that the operator $T$ is continuous at the point $x_{0}$. Since $x_{0}$ is an arbitrarily element chosen from $C(I), T$ is continuous on $C(I)$. On the other hand, for each $x \in C(I)$ and each $t \in I$ the inequality

$$
|(T x)(t)| \leq c+d\|x\|^{p}, \quad(p>0)
$$

is provided. Namely,

$$
\left|\frac{1+x^{2}(t)}{2}\right| \leq \frac{1}{2}+\frac{1}{2}\left|x^{2}(t)\right|=\frac{1}{2}+\frac{1}{2}|x(t)|^{2} \leq \frac{1}{2}+\frac{1}{2}\|x\|^{2}, \quad c=\frac{1}{2}, \quad d=\frac{1}{2}, \quad p=2 .
$$

There exists $r_{0}$ positive solution that provides the inequality

$$
a(\|\alpha\|)+\left(c+d r^{p}\right) M f(r) \leq r,
$$

where $\|\alpha\|=1, a(\|\alpha\|)=\frac{1}{5}, M=1$. Any number $r_{0}$ which provides the inequality

$$
0,375018 \leq r_{0} \leq 1,65394
$$

is a solution of the following inequality:

$$
\frac{1}{5}+\frac{1}{8}\left(1+e^{r}\right)\left(\frac{1}{2}+\frac{1}{2} r^{2}\right) \leq r
$$

For example $r_{0}=1$ is a solution of this inequality.

Let $X \neq \emptyset, X \subset B_{r_{0}}^{+}, \quad x \in B_{r_{0}}^{+}$and $t_{1}, t_{2} \in I$. We have the following estimate:

$$
\begin{aligned}
\left|\left(T x\left(t_{2}\right)\right)-\left(T x\left(t_{1}\right)\right)\right| & =\left|\frac{1+x^{2}\left(t_{2}\right)}{2}-\frac{1+x^{2}\left(t_{1}\right)}{2}\right| \\
& \leq \frac{1}{2}\left|x\left(t_{2}\right)+x\left(t_{1}\right)\right|\left|x\left(t_{2}\right)-x\left(t_{1}\right)\right| \\
& \leq \frac{1}{2}\left(\left|x\left(t_{2}\right)\right|+\left|x\left(t_{1}\right)\right|\right)\left|x\left(t_{2}\right)-x\left(t_{1}\right)\right| \\
& \leq \frac{1}{2}(\|x\|+\|x\|)\left|x\left(t_{2}\right)-x\left(t_{1}\right)\right| \\
& \leq \frac{1}{2}\left(2 r_{0}\right)\left|x\left(t_{2}\right)-x\left(t_{1}\right)\right|
\end{aligned}
$$




$$
\left.\left.\sup _{t_{1}, t_{2} \in I} \mid(T x)\left(t_{2}\right)\right)-(T x)\left(t_{1}\right)\right)\left|\leq \sup _{t_{1}, t_{2} \in I}\right| x\left(t_{2}\right)-x\left(t_{1}\right) \mid
$$

i.e.

$$
w(T x, \varepsilon) \leq w(x, \varepsilon)
$$

Thus, we have the following inequalities:

$$
\begin{gathered}
\sup _{x \in X} w(T x, \varepsilon) \leq \sup _{x \in X} w(x, \varepsilon), \\
w(T X, \varepsilon) \leq w(X, \varepsilon), \\
\lim _{\varepsilon \rightarrow 0} w(T X, \varepsilon) \leq \lim _{\varepsilon \rightarrow 0} w(X, \varepsilon), \\
w_{0}(T X) \leq w_{0}(X) .
\end{gathered}
$$

Let $X \neq \emptyset, \quad X \subset B_{r_{0}}^{+}, \quad x \in B_{r_{0}}^{+}, t_{1} \leq t_{2}$ and $t_{1}, t_{2} \in I$. In this case we have the following estimate:

$$
\begin{aligned}
& \left|(T x)\left(t_{2}\right)-(T x)\left(t_{1}\right)\right|-\left[(T x)\left(t_{2}\right)-(T x)\left(t_{1}\right)\right] \\
= & \left|\frac{1+x^{2}\left(t_{2}\right)-1-x^{2}\left(t_{1}\right)}{2}\right|-\left[\frac{1+x^{2}\left(t_{2}\right)-1-x^{2}\left(t_{1}\right)}{2}\right] \\
\leq & \frac{1}{2}\left|x\left(t_{2}\right)-x\left(t_{1}\right)\right|\left|x\left(t_{2}\right)+x\left(t_{1}\right)\right|-\frac{1}{2}\left[\left(x\left(t_{2}\right)-x\left(t_{1}\right)\right)\left(x\left(t_{2}\right)+x\left(t_{1}\right)\right)\right] \\
= & \frac{1}{2}\left(\left|x\left(t_{2}\right)\right|+\left|x\left(t_{1}\right)\right|\right)\left[\left|x\left(t_{2}\right)-x\left(t_{1}\right)\right|-\left(x\left(t_{2}\right)-x\left(t_{1}\right)\right)\right] \\
\leq & \frac{1}{2}(\|x\|+\|x\|)\left[\left|x\left(t_{2}\right)-x\left(t_{1}\right)\right|-\left(x\left(t_{2}\right)-x\left(t_{1}\right)\right)\right] \\
\leq & \frac{1}{2} 2 r_{0}\left[\left|x\left(t_{2}\right)-x\left(t_{1}\right)\right|-\left(x\left(t_{2}\right)-x\left(t_{1}\right)\right)\right] \\
= & \left|x\left(t_{2}\right)-x\left(t_{1}\right)\right|-\left[x\left(t_{2}\right)-x\left(t_{1}\right)\right] .
\end{aligned}
$$

Hence,

$$
\begin{aligned}
& \sup _{t_{1}, t_{2} \in I}\left[\left|(T x)\left(t_{2}\right)-(T x)\left(t_{1}\right)\right|-\left[(T x)\left(t_{2}\right)-(T x)\left(t_{1}\right)\right]\right] \\
& \leq \sup _{t_{1}, t_{2} \in I}\left[\left|x\left(t_{2}\right)-x\left(t_{1}\right)\right|-\left[x\left(t_{2}\right)-x\left(t_{1}\right)\right]\right], \\
& i(T x) \leq i(x)
\end{aligned}
$$

in view of the inequalities,

$$
\sup _{x \in X} i(T x) \leq \sup _{x \in X} i(x)
$$


and so, we obtain

$$
i(T X) \leq i(X) .
$$

From the inequalities (3.6) and (3.7), we get

$$
\mu(T X) \leq \mu(X),
$$

where $\theta$ can be taken as $\theta=1$. In this case the inequality $M f\left(r_{0}\right) \theta<1$ holds. Because, for $\theta=1, r_{0}=1, M=1$ and $f(1)=\frac{1+e}{8}$, the inequality

$$
M f(1) \theta=\frac{1+e}{8}<1
$$

holds. Since all of our assumptions are satisfied, this equation has a nondecreasing solution on $B_{r_{0}}^{+}$.

Remark 2. In the Example 3.1, since

$$
|(T x)(t)| \leq \frac{1}{2}+\frac{1}{2}\|x\|^{2}
$$

for all $x \in C(I)$ and $t \in I$, the condition $(v)$

$$
|(T x)(t)| \leq c+d\|x\|
$$

in [3] does not hold. Hence, the result given in [3] is not applicable to the integral equation (3.4) in the Example 3.1.

Example 3.2. Let us consider the equation

$$
x(t)=\frac{\sin \left(t-1+\frac{\pi}{2}\right)}{5}+\frac{1+x^{3}(t)}{7} \int_{0}^{t^{3}} \frac{\tan t+e^{x\left(\tau^{2}\right)}}{2+\tau} d \tau, t \in I=[0,1],
$$

where $\alpha(t)=t, \beta(t)=t, \gamma(t)=t^{3}, \eta(\tau)=\tau^{2}$, the function $a(t)=\frac{\sin \left(t-1+\frac{\pi}{2}\right)}{5}$ is nondecreasing and positive and $a(\|\alpha\|)=\frac{1}{5}$. We have the following estimate:

$$
|v(t, \tau, x)|=\left|\frac{\tan t+e^{x}}{2+\tau}\right| \leq \frac{\sqrt{3}+e^{x}}{2} \leq \frac{\sqrt{3}+e^{|x|}}{2}=f(|x|)
$$

for all $t, \tau \in I$ and $x \in \mathbb{R}$. From the above equation, we see that $f(x)=\frac{\sqrt{3}+e^{x}}{2}$ and $(T x)(t)=\frac{1+x^{3}(t)}{7}$. It is obvious that $T: C(I) \rightarrow C(I)$. Let us see that the operator $T$ is continuous. Let $x_{0}$ be an arbitrarily element chosen from $C(I)$. When $\left\|x-x_{0}\right\|<\delta$ we have the following estimate:

$$
\begin{aligned}
\left\|T x-T x_{0}\right\| & =\max _{t \in I}\left|\frac{1+x^{3}(t)}{7}-\frac{1+x_{0}^{3}(t)}{7}\right| \\
& =\frac{1}{7} \max _{t \in I}\left|x^{3}(t)-x_{0}^{3}(t)\right| \\
& =\frac{1}{7} \max _{t \in I}\left[\left|x(t)-x_{0}(t) \| x^{2}(t)+x(t) x_{0}(t)+x_{0}^{2}(t)\right|\right]
\end{aligned}
$$




$$
\begin{aligned}
\left|x^{2}(t)+x(t) x_{0}(t)+x_{0}^{2}(t)\right| & =\left|\left(x(t)-x_{0}(t)\right)^{2}+3 x(t) x_{0}(t)\right| \\
& \leq\left|\left(x(t)-x_{0}(t)\right)\right|^{2}+3\left|x(t) x_{0}(t)\right| \\
& <\delta^{2}+3\left|x(t)-x_{0}(t)+x_{0}(t)\right|\left|x_{0}(t)\right| \\
& \leq \delta^{2}+3 \delta\left\|x_{0}\right\|+3\left\|x_{0}\right\|^{2} .
\end{aligned}
$$

From the above inequalities, we obtain

$$
\begin{aligned}
\left\|T x-T x_{0}\right\| & =\frac{1}{7} \max _{t \in I}\left[\left|x(t)-x_{0}(t) \| x^{2}(t)+x(t) x_{0}(t)+x_{0}^{2}(t)\right|\right] \\
& <\frac{1}{7}\left(\delta^{3}+3 \delta^{2}\left\|x_{0}\right\|+3 \delta\left\|x_{0}\right\|^{2}\right) \\
& =\frac{1}{7}\left(\left(\delta+\left\|x_{0}\right\|\right)^{3}-\left\|x_{0}\right\|^{3}\right) \\
& =\frac{1}{7}\left(\delta+\left\|x_{0}\right\|\right)^{3}-\frac{1}{7}\left\|x_{0}\right\|^{3}=\varepsilon \\
& \Rightarrow \frac{1}{7}\left(\delta+\left\|x_{0}\right\|\right)^{3}=\varepsilon+\frac{1}{7}\left\|x_{0}\right\|^{3} \\
& \Rightarrow \delta+\left\|x_{0}\right\|=\left(7 \varepsilon+\left\|x_{0}\right\|^{3}\right)^{\frac{1}{3}} \\
& \Rightarrow \delta=\left(7 \varepsilon+\left\|x_{0}\right\|^{3}\right)^{\frac{1}{3}}-\left\|x_{0}\right\|>0 .
\end{aligned}
$$

If $\delta$ is chosen as $\delta=\left(7 \varepsilon+\left\|x_{0}\right\|^{3}\right)^{\frac{1}{3}}-\left\|x_{0}\right\|>0$, it is seen that the operator $T$ is continuous at the point $x_{0}$. Since $x_{0}$ is an arbitrarily element chosen from $C(I), T$ is continuous on $C(I)$. Since

$$
\begin{gathered}
|(T x)(t)|=\left|\frac{1+x^{3}(t)}{7}\right| \leq \frac{1}{7}+\frac{1}{7}\left|x^{3}(t)\right|=\frac{1}{7}+\frac{1}{7}|x(t)|^{3} \leq \frac{1}{7}+\frac{1}{7}\|x\|^{3}, \\
c=\frac{1}{7}, \quad d=\frac{1}{7}, \quad p=3,
\end{gathered}
$$

the inequality

$$
|(T x)(t)| \leq c+d\|x\|^{p}, \quad(p>0)
$$

holds. There exists positive solution $r_{0}$ that provides the inequality

$$
a(\|\alpha\|)+\left(c+d r^{p}\right) M f(r) \leq r,
$$

where $\|\alpha\|=1, a(\|\alpha\|)=\frac{1}{5}, M=1$.

Any number $r_{0}$ providing the inequality

$$
0,386812 \leq r_{0} \leq 1,32116
$$

is a solution of the following inequality:

$$
\left(\frac{1}{2}\left(\sqrt{3}+e^{r}\right)\right)\left(\frac{1}{7}+\frac{1}{7} r^{3}\right)+\frac{1}{5} \leq r .
$$


For example $r_{0}=1$ is a solution of this inequality.

For any $t_{1}, t_{2} \in[0,1]$ such that $\left|t_{2}-t_{1}\right| \leq \varepsilon, \emptyset \neq X \subset B_{r_{0}}^{+}=B_{1}^{+}$and $x \in X$, we obtain

$$
\begin{aligned}
\left|(T x)\left(t_{2}\right)-(T x)\left(t_{1}\right)\right| & =\left|\frac{1+x^{3}\left(t_{2}\right)}{7}-\frac{1+x^{3}\left(t_{1}\right)}{7}\right| \\
& =\frac{1}{7}\left|x^{3}\left(t_{2}\right)-x^{3}\left(t_{1}\right)\right| \\
& \leq \frac{1}{7}\left|x\left(t_{2}\right)-x\left(t_{1}\right)\right|\left|x^{2}\left(t_{2}\right)+x\left(t_{2}\right) x\left(t_{1}\right)+x^{2}\left(t_{1}\right)\right| \\
& \leq \frac{1}{7}\left|x\left(t_{2}\right)-x\left(t_{1}\right)\right|\left(\left|x^{2}\left(t_{2}\right)\right|+\left|x\left(t_{2}\right) x\left(t_{1}\right)\right|+\left|x^{2}\left(t_{1}\right)\right|\right) \\
& \leq \frac{1}{7}\left|x\left(t_{2}\right)-x\left(t_{1}\right)\right| 3|| x \|^{2} \\
& \leq \frac{3}{7}\left|x\left(t_{2}\right)-x\left(t_{1}\right)\right| .
\end{aligned}
$$

If we take the supremum on both sides of inequality over $t_{1}, t_{2} \in I$ such that $\left|t_{2}-t_{1}\right| \leq \varepsilon$ and $x \in X$, we get

$$
w(T x, \varepsilon) \leq \frac{3}{7} w(x, \varepsilon) .
$$

If we take the supremum at this inequality over $x \in X$, we get

$$
w(T X, \varepsilon) \leq \frac{3}{7} w(X, \varepsilon),
$$

where, for $\varepsilon \rightarrow 0$, we obtain

$$
w_{0}(T X) \leq \frac{3}{7} w_{0}(X)
$$

For any $t_{1}, t_{2} \in[0,1]$ such that $t_{1} \leq t_{2}, \emptyset \neq X \subset B_{r_{0}}^{+}=B_{1}^{+}$and $x \in X$, we get

$$
\begin{aligned}
& \left|(T x)\left(t_{2}\right)-(T x)\left(t_{1}\right)\right|-\left[(T x)\left(t_{2}\right)-(T x)\left(t_{1}\right)\right] \\
= & \left|\frac{1+x^{3}\left(t_{2}\right)}{7}-\frac{1+x^{3}\left(t_{1}\right)}{7}\right|-\left[\frac{1+x^{3}\left(t_{2}\right)}{7}-\frac{1+x^{3}\left(t_{1}\right)}{7}\right] \\
= & \frac{1}{7}\left|x^{3}\left(t_{2}\right)-x^{3}\left(t_{1}\right)\right|-\frac{1}{7}\left[x^{3}\left(t_{2}\right)-x^{3}\left(t_{1}\right)\right] \\
= & \frac{1}{7}\left|\left(x\left(t_{2}\right)-x\left(t_{1}\right)\right)\left(x^{2}\left(t_{2}\right)+x\left(t_{2}\right) x\left(t_{1}\right)+x^{2}\left(t_{1}\right)\right)\right| \\
- & \frac{1}{7}\left(x\left(t_{2}\right)-x\left(t_{1}\right)\right)\left(x^{2}\left(t_{2}\right)+x\left(t_{2}\right) x\left(t_{1}\right)+x^{2}\left(t_{1}\right)\right) \\
= & \frac{1}{7}\left(x^{2}\left(t_{2}\right)+x\left(t_{2}\right) x\left(t_{1}\right)+x^{2}\left(t_{1}\right)\right)\left[\left|x\left(t_{2}\right)-x\left(t_{1}\right)\right|-\left(x\left(t_{2}\right)-x\left(t_{1}\right)\right)\right] \\
\leq & \frac{3}{7}\|x\|^{2}\left[\left|x\left(t_{2}\right)-x\left(t_{1}\right)\right|-\left(x\left(t_{2}\right)-x\left(t_{1}\right)\right)\right] \\
\leq & \frac{3}{7}\left[\left|x\left(t_{2}\right)-x\left(t_{1}\right)\right|-\left(x\left(t_{2}\right)-x\left(t_{1}\right)\right)\right] .
\end{aligned}
$$


If we take the supremum at this inequality over $t_{1}, t_{2} \in[0,1]$ such that $t_{1} \leq t_{2}$, we get

$$
i(T x) \leq \frac{3}{7} i(x) .
$$

If we take the supremum at this inequality over $x \in X$, we get

$$
i(T X) \leq \frac{3}{7} i(X) .
$$

From the inequalities (3.9) and (3.10) we get

$$
\mu(T X) \leq \frac{3}{7} \mu(X) .
$$

Then, $\theta$ can be taken as $\theta=\frac{3}{7}$. For $r=1, \theta=\frac{3}{7}$ and $M=1$, we get

$$
M f(1) \frac{3}{7}<1 \text {. }
$$

Thus, all of our assumptions provide and hence this equation has a nondecreasing solution on $B_{r_{0}}^{+}$.

Remark 3. In the Example 3.2, since

$$
|(T x)(t)| \leq \frac{1}{7}+\frac{1}{7}\|x\|^{3}
$$

for all $x \in C(I)$ and $t \in I$, the condition $(v)$

$$
|(T x)(t)| \leq c+d\|x\|
$$

in [3] does not hold. Hence, the result given in [3] is not applicable to the integral equation (3.8) presented in the Example 3.2.

\section{Acknowledgments}

The authors would like to thanks the referees for their suggestions and corrections.

\section{References}

[1] R.P. Agarwal, D. O'Regan, P.J.Y. Wong, Positive Solutions of Differential and Integral Equations, Kluwer Academic Publishers, Dordrecht, 1999.

[2] I.K. Argyros, Quadratic equations applications to Chandrasekhar's and related equations, Bull. Austral. Math. Soc. 32 (1985) 275-292.

[3] J. Banaś, J. Caballero, J. Rocha, K. Sadarangani, Monotonic solutions of a class of quadratic integral equations of Volterra type, Comput. Math. Applic. 49 (2005) 943-952. 
[4] J. Banaś, K. Goebel, Measures of Noncompactness in Banach Spaces, Marcel Dekker, New York, 1980.

[5] J. Banaś, A. Martinon, On monotonic solutions of a quadratic integral equation of Volterra type, Comput. Math. Applic. 47 (2004) 271-279.

[6] J. Banaś, L. Olszowy, Measure of noncompactness related to monotoncity, Comment. Math. 41 (2001) 13-23.

[7] J. Banaś, K. Sadarangani, Solvabolity of Volterra-Stieltjes operator-integral equations and their applications, Comput. Math. Applic. 41 (12) (2001) 1535-1544.

[8] G. Darbo, Punti uniti in trasformazioni a codominio non compatto, Rend. Sem. Mat. Univ. Padova 24 (1955) 84-92.

[9] K. Deimling, Nonlinear Functional Analysis, Springer-Verlag, Berlin, 1985.

[10] S. Hu, M. Khavanin, W. Zhuang, Integral equations arising in the kinetic theory of gases, Appl. Analysis 34 (1989) 261-266.

[11] D. O'Regan, M.M. Meehan, Existence Theory for Nonlinear Integral and Integrodifferential Equations, Kluwer Academic Publishers, Dordrecht, 1998.

\section{DOI: $10.7862 / \mathrm{rf} .2019 .8$}

\section{Osman Karakurt}

email: osman-44@yandex.com

ORCID: 0000-0002-4669-8470

Yeşilyurt G. N. Mesleki ve Teknik Anadolu Lisesi

Malatya

TURKEY

\section{Ömer Faruk Temizer*}

email: omer.temizer@inonu.edu.tr

ORCID: 0000-0002-3843-5945

Eğitim Fakültesi, A-Blok

İnönü Üniversitesi

44280-Malatya

TURKEY

${ }^{*}$ Corresponding author

Received 10.04.2019 